\title{
RegPrecise 3.0 - A resource for genome-scale exploration of transcriptional regulation in bacteria
}

\author{
Pavel S Novichkov ${ }^{1 *}$, Alexey E Kazakov ${ }^{1}$, Dmitry A Ravcheev ${ }^{2,3}$, Semen A Leyn², Galina Y Kovaleva ${ }^{1,2}$, \\ Roman A Sutormin ${ }^{1,4}$, Marat D Kazanov ${ }^{2}$, William Riehl', Adam P Arkin ${ }^{1}$, Inna Dubchak ${ }^{1}$ and Dmitry A Rodionov ${ }^{2,3^{*}}$
}

\begin{abstract}
Background: Genome-scale prediction of gene regulation and reconstruction of transcriptional regulatory networks in prokaryotes is one of the critical tasks of modern genomics. Bacteria from different taxonomic groups, whose lifestyles and natural environments are substantially different, possess highly diverged transcriptional regulatory networks. The comparative genomics approaches are useful for in silico reconstruction of bacterial regulons and networks operated by both transcription factors (TFs) and RNA regulatory elements (riboswitches).

Description: RegPrecise (http://regprecise.lbl.gov) is a web resource for collection, visualization and analysis of transcriptional regulons reconstructed by comparative genomics. We significantly expanded a reference collection of manually curated regulons we introduced earlier. RegPrecise 3.0 provides access to inferred regulatory interactions organized by phylogenetic, structural and functional properties. Taxonomy-specific collections include 781 TF regulogs inferred in more than 160 genomes representing 14 taxonomic groups of Bacteria. TF-specific collections include regulogs for a selected subset of 40 TFs reconstructed across more than 30 taxonomic lineages. Novel collections of regulons operated by RNA regulatory elements (riboswitches) include near 400 regulogs inferred in 24 bacterial lineages. RegPrecise 3.0 provides four classifications of the reference regulons implemented as controlled vocabularies: 55 TF protein families; 43 RNA motif families; 150 biological processes or metabolic pathways; and 200 effectors or environmental signals. Genome-wide visualization of regulatory networks and metabolic pathways covered by the reference regulons are available for all studied genomes. A separate section of RegPrecise 3.0 contains draft regulatory networks in 640 genomes obtained by an conservative propagation of the reference regulons to closely related genomes.

Conclusions: RegPrecise 3.0 gives access to the transcriptional regulons reconstructed in bacterial genomes. Analytical capabilities include exploration of: regulon content, structure and function; TF binding site motifs; conservation and variations in genome-wide regulatory networks across all taxonomic groups of Bacteria. RegPrecise 3.0 was selected as a core resource on transcriptional regulation of the Department of Energy Systems Biology Knowledgebase, an emerging software and data environment designed to enable researchers to collaboratively generate, test and share new hypotheses about gene and protein functions, perform large-scale analyses, and model interactions in microbes, plants, and their communities.
\end{abstract}

Keywords: Regulatory network, Regulon, Transcription factor, Riboswitch, Comparative genomics, Bacteria

\footnotetext{
* Correspondence: PSNovichkov@lbl.gov; rodionov@burnham.org

${ }^{1}$ Lawrence Berkeley National Laboratory, Berkeley 94710, CA, USA

${ }^{2}$ A.A. Kharkevich Institute for Information Transmission Problems, Russian

Academy of Sciences, Moscow 127994, Russia

Full list of author information is available at the end of the article
} 


\section{Background}

Fine-tuned regulation of gene transcription in response to extracellular and intracellular signals is a key mechanism for successful adaptation of microorganisms to changing environmental conditions. Activation and repression of gene expression in bacteria is usually mediated by DNA-binding transcription factors (TFs) that specifically recognize TF-binding sites (TFBSs) in upstream regions of target genes, and also by various regulatory RNA structures including cis-acting metabolite-sensing riboswitches and attenuators encoded in the leader regions of target genes. Genes and operons directly coregulated by the same TF (or by RNA motifs from the same structural family) form a so called regulon [1]. All regulons together operated in the same genome form a transcriptional regulatory network (TRN) of a cell.

Computational methods based on the comparison of TFBSs in related species proved to be efficient for predicting transcriptional regulons in Bacteria [2-5]. To address the challenge of regulatory network reconstruction in ever growing number of sequenced microbial genomes, we recently developed a strategy for fast and accurate comparative reconstruction of large-scale TRNs and implemented it in the RegPredict web server [6]. First, the bacterial species tree is subdivided into small taxonomic groups, and a subset of 5-15 representative genomes from each group is selected. Second, semiautomatic reconstruction of reference regulogs (orthologous regulons) in these selected genomes is carried out using both known TF-binding motifs and ab initio predicted novel DNA motifs (reviewed in [1]). Resulting regulons are characterized by a TF, predicted DNAbinding motif, and a set of target genes/operons together with associated TFBSs in their upstream regions. A regulog, that is a group of regulons operated by the orthologous TFs in closely related genomes, represents the main outcome of the RegPredict-based analysis. The reference regulogs are then used for an automatic propagation of the captured regulatory interactions into new genomes from the same taxonomic group.

By applying this computational approach to a growing number of complete bacterial genomes, we inferred highquality genome-scale TRNs for diverse taxonomic groups of bacteria, namely Shewanella, Thermotoga, Desulfovibrio, Bacillus, Lactobacillus, Streptococcus and Staphylococcus spp. [7-20]. To provide public access to the collections of transcriptional regulons reconstructed via the RegPredict web server, some time ago we had developed the first version of the RegPrecise database for capturing and visualization of the curated regulon inferences [21]. Recently added RegPrecise web services [22] allow for programmatic access to the transcriptional regulatory data.

Here we present RegPrecise Version 3.0 with significantly increased biological data content and novel database features. The current database contains more than 1500 regulogs including 400 regulogs controlled by RNA regulatory motifs in 24 taxonomic groups, and $\sim 800$ TF-operated regulogs in 14 taxonomic collections. Novel features of RegPrecise 3.0 include controlled vocabularies for effectors and metabolic pathways, detailed classifications for TF proteins and RNA motif families, and several types of visualization for genome-wide regulatory networks. RegPrecise 3.0 is a largest and fast growing web resource for comparative genomics of transcriptional regulation in Bacteria. It is highly valuable both for experimental biologists studying mechanisms of transcriptional regulation in bacteria, and computational biologists interested in modeling metabolic and regulatory networks.

\section{Construction and content}

The RegPrecise database contains detailed information on regulatory interactions and transcriptional regulons inferred by a comparative genomics in diverse bacterial genomes [21]. In addition to TF-operated regulons, the updated version of the database includes the inferred regulons for RNA regulatory motifs (riboswitches) [23]. Below we describe the database structure and data organization, and present new features and statistics for significantly updated RegPrecise 3.0 content.

The database has the following hierarchical data organization: (i) a regulon; (ii) a regulog; and (iii) a collection of regulogs (Figure 1). A regulon is a basic unit of the database that represents a set of target genes/operons that are co-regulated by the same regulator (TF or RNA motif) in a particular genome. The description of each regulon in RegPrecise also includes an alignment of TF binding sites (or RNA regulatory sites). A regulog represents a set of regulons under control of orthologous regulators in a group of taxonomically related genomes. Each TF-operated regulog has a TFBS motif represented as a sequence logo or an alignment.

Our strategy for regulon reconstruction in RegPrecise includes four steps (Figure 2): (i) selection of a group of closely-related bacteria on the species tree; (ii) selection of a subset of diverse genomes that represent a given taxonomic group; (iii) reconstruction and manual curation of reference regulogs in the selected genomes; (iv) accurate automatic propagation of the reference regulogs to the large set of closely-related genomes from the same taxonomic group. Accordingly, the RegPrecise 3.0 database includes two major sections: (1) reference regulog collections, and (2) propagated regulons. Below we describe data construction and content for each of these sections.

\section{Building reference regulog collections}

We use RegPredict web server [6] for careful comparative analysis and manual curation of each regulog in 


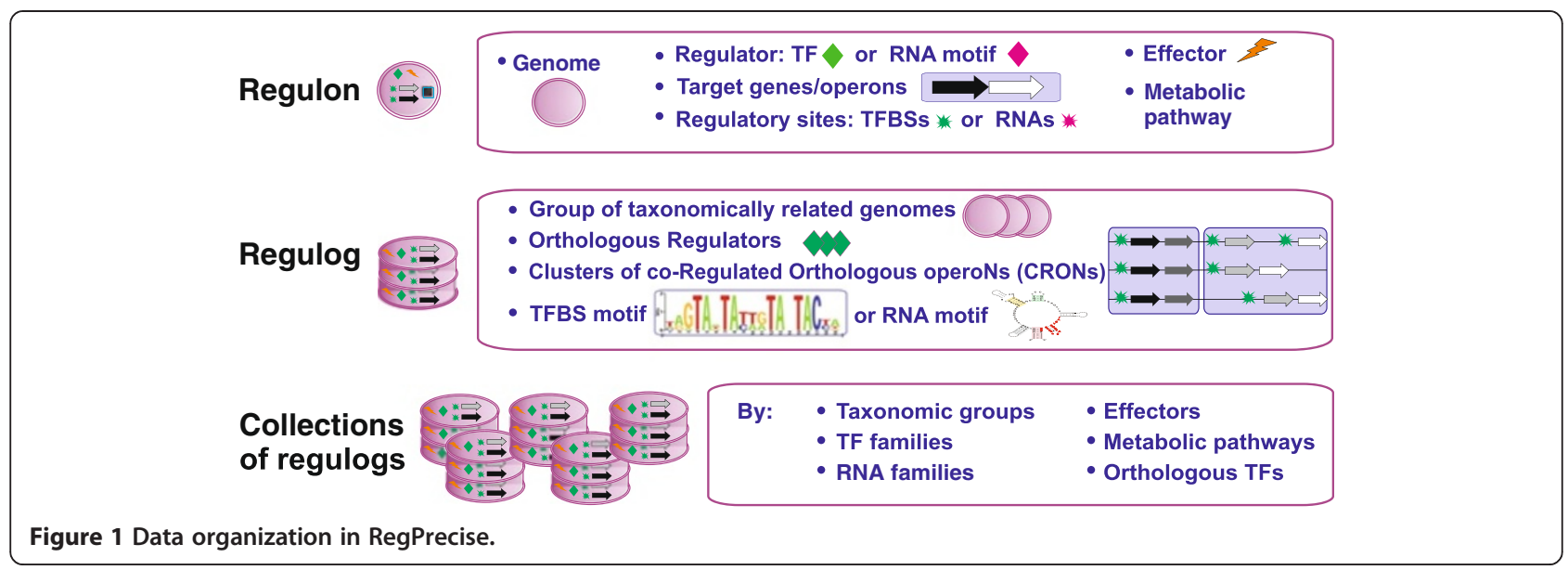

RegPrecise. RegPredict allows for the simultaneous analysis of multiple microbial genomes and integrates information on gene orthologs, operon predictions, and functional gene annotations. It implements two wellestablished workflows for inference of TF-operated regulons: i) regulon reconstruction for known TFBS motifs, and ii) ab initio inference of novel TFBS motifs and regulons. For experimentally characterized regulons, we used training sets of known TFBSs collected from literature and other regulatory databases [24-27] to build a position weight matrix (PWM) for a TFBS motif. Novel TFBS motifs were identified by Discover profile tool of RegPredict using sets of potentially co-regulated genes. Constructed PWMs for DNA motifs (both known and ab initio predicted) were used to scan each selected genome and identify genes with candidate regulatory sites in upstream regions. Each predicted regulatory interaction was analyzed for conservation across the group of closely related genomes using the Clusters of co-Regulated Orthologous operoNs (CRONs) in RegPredict. For each analyzed regulon, the set of constructed CRONs was prioritized based on the level of conservation of regulatory interactions, emphasizing the most prominent regulon members. At the next step, we conducted the functional and genomic context analysis of each CRON using the advanced web interface facilitating the decision on CRON inclusion in the final regulog model. Combining all accepted CRONs for a given TFBS motif produces the reconstructed TF regulog for a group of target genomes.

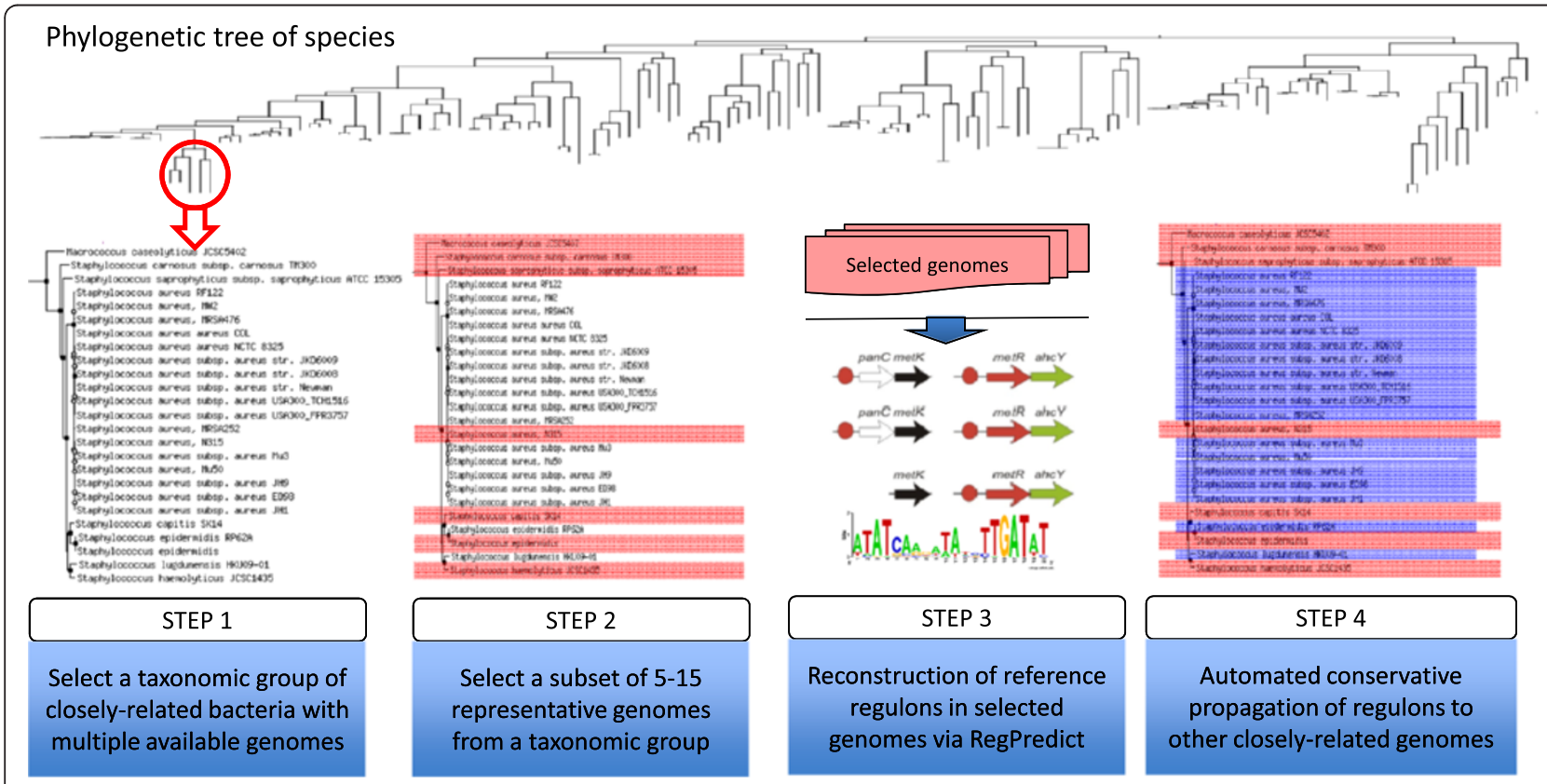

Figure 2 Comparative genomics-driven strategy for regulon inference in RegPrecise. 
Table 1 Taxonomic collections of curated genome-wide TRNs in diverse microbes

\begin{tabular}{|c|c|c|c|c|c|c|c|c|}
\hline Taxonomic group & Phylum & $\begin{array}{l}\text { Reference } \\
\text { genomes }\end{array}$ & TF regulogs & RNA regulogs & $\begin{array}{l}\text { TF binding } \\
\text { sites }\end{array}$ & RNA sites & $\begin{array}{l}\text { Regulated } \\
\text { genes }^{1}\end{array}$ & $\begin{array}{l}\text { Genes per } \\
\text { genome }^{2}\end{array}$ \\
\hline Bacillales & Firmicutes & 11 & 134 & 39 & 3815 & 668 & 7301 & 664 \\
\hline Staphylococcus & Firmicutes & 7 & 48 & 29 & 1965 & 288 & 3329 & 476 \\
\hline Lactobacillaceae & Firmicutes & 15 & 79 & 39 & 1811 & 581 & 3784 & 252 \\
\hline Streptococcaceae & Firmicutes & 15 & 69 & 29 & 3118 & 400 & 5652 & 377 \\
\hline Clostridiaceae & Firmicutes & 20 & 7 & 40 & 303 & 968 & 2489 & 124 \\
\hline Enterobacteriales & Proteobacteria & 12 & 87 & 18 & 7365 & 188 & 9028 & 752 \\
\hline Shewanella & Proteobacteria & 16 & 80 & 15 & 8450 & 291 & 10817 & 676 \\
\hline Ralstonia & Proteobacteria & 6 & 24 & 10 & 574 & 66 & 1297 & 216 \\
\hline Desulfovibrionales & Proteobacteria & 10 & 92 & 9 & 1942 & 72 & 3368 & 337 \\
\hline Thermotogales & Thermotogae & 11 & 33 & 13 & 642 & 88 & 2153 & 196 \\
\hline Corynebacteriaceae & Actinobacteria & 8 & 45 & 13 & 937 & 80 & 1624 & 203 \\
\hline Bacteroidaceae & Bacteroidae & 11 & 35 & 2 & 667 & 84 & 1797 & 163 \\
\hline Chloroflexi & Chloroflexi & 5 & 30 & 17 & 314 & 98 & 1014 & 203 \\
\hline Cyanobacteria & Cyanobacteria & 14 & 18 & 11 & 1032 & 86 & 1442 & 103 \\
\hline Total: & - & 161 & 781 & 284 & 32935 & 3958 & 55095 & 342 \\
\hline
\end{tabular}

${ }^{1}$ Total number of regulated genes in all TF- and RNA operated regulons.

${ }^{2}$ Average numbers of regulated genes per genome were calculated for each studied taxonomic group; the average numbers for all lineages are given in the last line.

We utilized a similar workflow for reconstruction of regulogs operated by RNA motifs. First, RNA regulatory sites were identified in the studied genomes using the probabilistic covariance models for 43 RNA families from the Rfam database [28] and the Infernal program [29]. Then, the identified candidate RNA sites were uploaded into RegPredict and used for regulog reconstruction using the similar CRON-based approach as for TF regulogs. Thus, each inferred RNA regulog includes all genes/operons that are preceded by a candidate Rfam-family motif in a studied taxonomic group of genomes [23].

\section{Collections of regulogs}

All reference regulogs are classified into collections of six biological types briefly described below.

Taxonomic collections represent results of large-scale reconstructions of both TF- and RNA-operated regulogs

Table 2 Collections of TF regulons reconstructed by conservative propagation

\begin{tabular}{|c|c|c|c|c|c|c|}
\hline \multicolumn{3}{|c|}{ Propagated regulons } & \multicolumn{4}{|c|}{ Reference regulons } \\
\hline Taxonomic group & Genomes & TF regulons & Regulog collection & Genomes & TF regulogs & TF regulons \\
\hline Bacillales & 68 & 3784 & Bacillales & 11 & 134 & 844 \\
\hline Staphylococcaceae & 25 & 876 & Staphylococcus & 7 & 48 & 271 \\
\hline Lactobacillaceae & 29 & 873 & Lactobacillaceae & 15 & 79 & 483 \\
\hline Streptococcaceae & 69 & 2644 & Streptococcaceae & 15 & 69 & 593 \\
\hline Clostridia & 61 & 144 & Clostridiaceae & 20 & 7 & 51 \\
\hline Enterobacteriales & 160 & 7735 & Enterobacteriales & 12 & 87 & 698 \\
\hline Alteromonadales & 39 & 1444 & Shewanella & 16 & 80 & 862 \\
\hline Burkholderiaceae & 74 & 1022 & Ralstonia & 6 & 24 & 122 \\
\hline Desulfovibrionales & 11 & 349 & Desulfovibrionales & 10 & 92 & 392 \\
\hline Thermotogales & 11 & 223 & Thermotogales & 11 & 33 & 239 \\
\hline Corynebacteriaceae & 9 & 237 & Corynebacteriaceae & 8 & 45 & 209 \\
\hline Bacteroidaceae & 22 & 254 & Bacteroidaceae & 11 & 35 & 215 \\
\hline Chloroflexi & 14 & 139 & Chloroflexi & 5 & 30 & 107 \\
\hline Cyanobacteria & 48 & 510 & Cyanobacteria & 14 & 18 & 180 \\
\hline Total: & 640 & 20234 & Total: & 161 & 781 & 5266 \\
\hline
\end{tabular}


in narrow taxonomic groups of bacteria. RegPrecise 3.0 contains 14 taxonomic collections covering major phyla of Bacteria and including 781 regulogs (Table 1). These data represent a major expansion since the 1.0 version that had only two taxonomic collections [21]. The reconstructed genome-wide TRNs for bacteria from six taxonomic collections (Shewanella, Staphylococcus, Bacillales, Streptococcaceae, Lactobacillaceae and Thermotogales) have been described in our research papers $[10,12,13,19,20]$, whereas publications on other taxonomic collections are currently in preparation. The reconstructed genome-specific TRNs utilize and expand experimental knowledge on regulatory interactions accumulated in the RegTransBase database developed in our group [25], and other specialized databases (DBTBS [27], RegulonDB [26], CoryneRegNet [24]). For instance, the Bacillus subtilis TRN was expanded by $\sim 300$ new target genes and 36 novel TF regulons that await future experimental validation [10]. The genome-wide TRNs from taxonomy collections are useful for building predictive metabolic models with regulatory constraints.

TF collections contain regulogs for a selected subset of TFs conserved in more than three taxonomic groups. Each TF collection represents all reconstructed regulogs for a given set of orthologous TFs across different taxonomic groups of bacteria. The RegPrecise 3.0 contains 40 TF collections (an increase of 31 TFs since the previous database version) that include both widespread TFs such as NrdR, LexA and Zur present in more than 25 diverse taxonomic groups, and narrowly distributed TFs such as Irr in $\alpha$-proteobacteria and PurR in $\gamma$-proteobacteria. Altogether, the orthologous TF collections include 443 regulogs that are valuable for comparative and evolutionary analysis of TF binding motifs and regulon contents, as illustrated by our previous publications on comparative genomics analyses of numerous TFs including HexR [11],

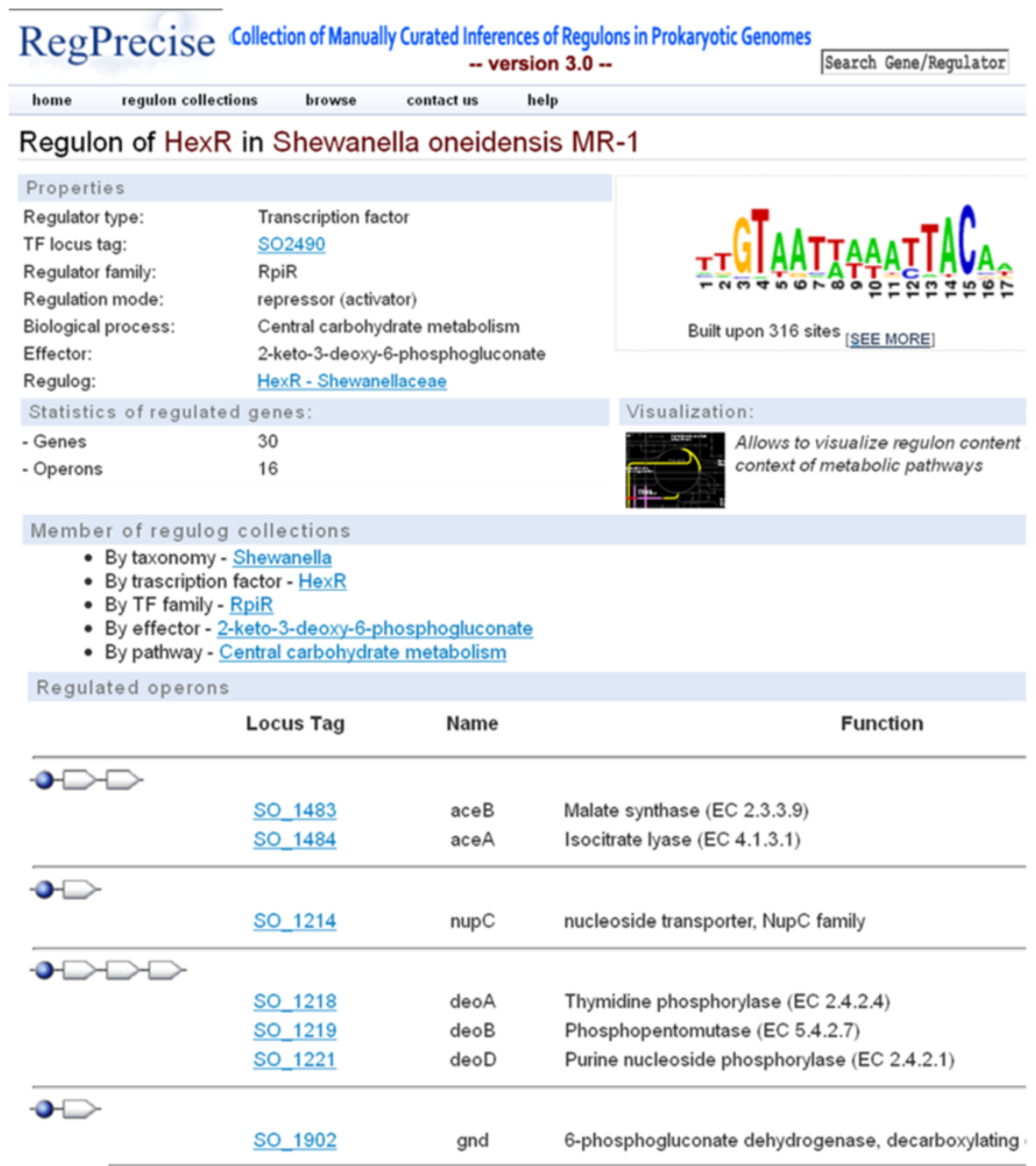

Figure 3 The regulon page in RegPrecise. The screenshot illustrates the details of the HexR regulon in Shewanella oneidensis. 
Rex [14], NrdR [17], NrtR [30], NiaR [31], KdgR and ExuR [32], AraR and XylR [33], PsrA and LiuR [7], NsrR and NorR [16], Irr and IscR [18], BirA [34], and PaaR [35].

TF family collections provide structural classification of more than 7000 TFs that belong to more than 1000 reconstructed TF regulogs. All studied TFs were classified into 55 protein families based on their domain composition in the Pfam [36], COG [37] and Superfamily [38] libraries (see TF families classification and domains architecture in Additional file 1). Annotations of TF protein domains were collected from the MicrobesOnline database [39]. Each TF family was characterized by at least one DNA-binding domain and one or several additional domains involved in effector sensing and/or dimerization. In RegPrecise 3.0, we provide a short summary with literature citations for each of the $55 \mathrm{TF}$ families. The TF family collections covers both large and diverse families such as LacI, GntR, and TetR that contain more than $100 \mathrm{TF}$ regulogs, and narrow families such as ArgR, BirA and LexA containing orthologous TFs of the same function. These collections are valuable for evolutionary analysis of TF binding site motifs and effector specificities within the same TF family.
RNA family collections is a novel section that provides an access to near 400 reconstructed regulogs operated by the RNA regulatory elements in more than 250 bacterial genomes from 24 taxonomic groups. The RegPrecise 3.0 includes RNA family collections for 43 Rfam families. For each collection we provide a short biological summary with literature citations and cross references to the Rfam database [28]. Among the analyzed regulatory RNAs are 15 metabolite-sensing riboswitches, 6 ribosomal operon leaders, 4 amino acid-responsive attenuators, and multiple cis-acting regulatory RNAs of yet unknown regulatory mechanisms. The large collection of T-box regulogs for amino acid metabolism was additionally classified by amino acid specificities of T-boxes deduced from their multiple sequence alignment. The detailed evolutionary analysis of regulog content from the RNA family collections was recently published [23].

Effector and Pathway collections represent two novel functional classifications of regulogs in RegPrecise 3.0. We used controlled vocabularies of 255 regulatory effectors and 235 metabolic pathways to organize collections of these two types. Effectors were retrieved from manually curated annotations of TF- and RNA-operated regulogs in

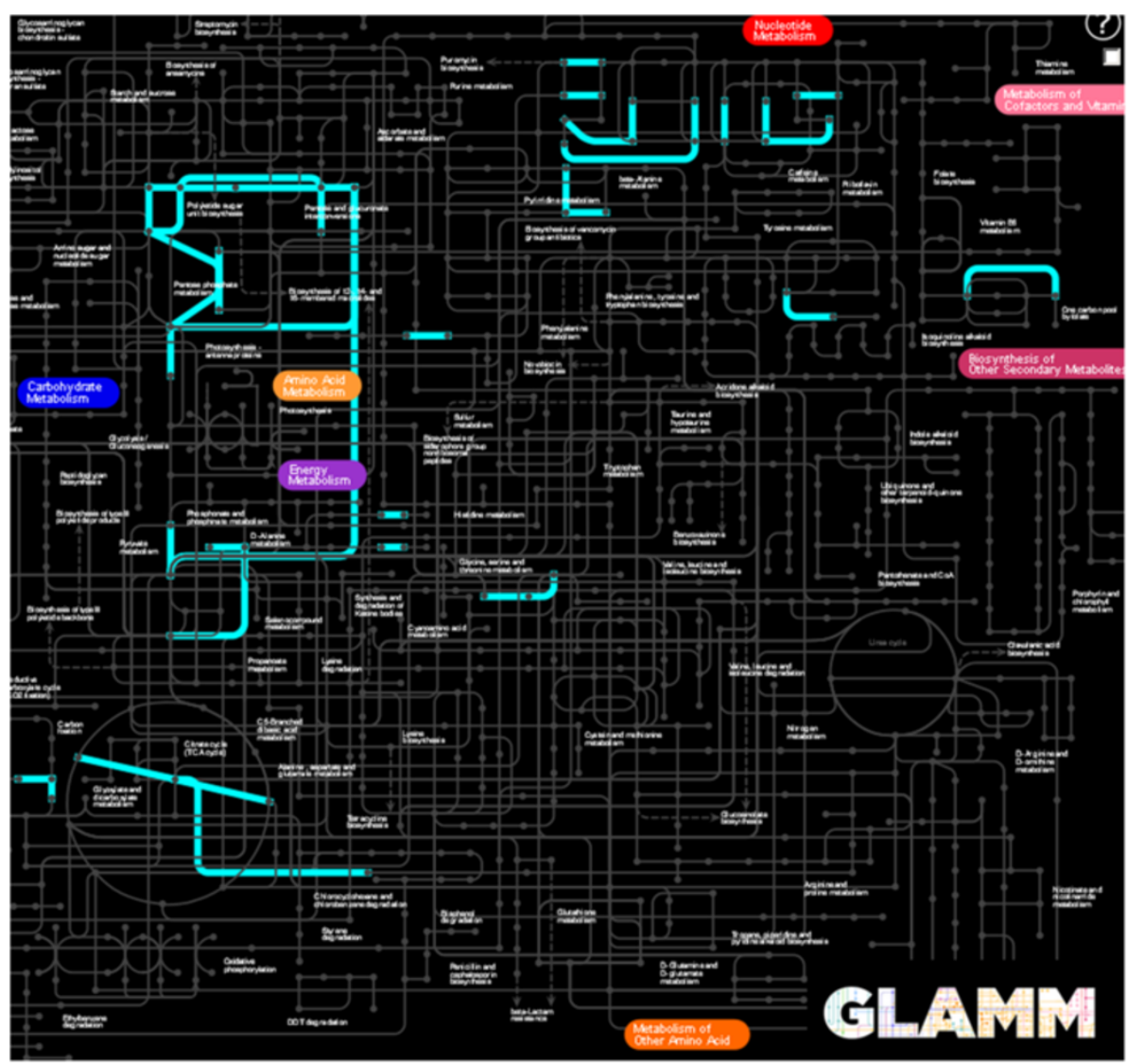

Figure 4 A GLAMM representation of functional regulon content in RegPrecise. The screenshot highlights metabolic pathways and reactions controlled by the HexR regulon in Shewanella oneidensis. 
RegPrecise, and assigned to 12 higher-level categories. These categories include amino acids, aminoacyl-tRNAs, antibiotics, carbohydrates, coenzymes, heterocyclic compounds, inorganic chemicals, lipids and fatty acids, nucleotides and nucleosides, organic chemicals, peptides and proteins and other factors (according to $\mathrm{MeSH}$ headings). Metabolic pathways and biological processes were assigned to regulogs based on the analysis of functional regulon content and experimental data from the literature. The RegPrecise 3.0 contains 235 pathways classified into 23 functional categories according to the biological subsystems classification from the SEED database [40]. Two largest functional categories of regulogs in RegPrecise are those involved in the metabolism of carbohydrates and amino acids.

\section{Propagated TF regulons}

The obtained reference TF regulogs were used for largescale annotation of regulatory interactions in closely related genomes by using an automated conservative propagation procedure. For each taxonomic collection including manually curated regulons in the selected subset of genomes, we selected an expanded set of genomes from the same taxonomic lineage that are available in the MicrobesOnline database [39]. To propagate a particular TF regulog to a target genome, we identified orthologs for both a TF gene and each of the previously described members of a reference TF regulog using the pre-computed ortholog groups in MicrobesOnline. For the identified gene orthologs in target genomes, we perform search for candidate TFBSs in their upstream regions (from -350 to +50 bp relative to the start codon, excluding the coding regions of upstream genes). For search of putative binding sites we utilized a PWM that is associated with the reference regulog and was used for its original reconstruction. Each propagated regulon has one or more candidate regulated genes, their upstream binding sites, and, in most cases, an attributed orthologous TF. Moreover, we explicitly provide comparative genomics evidences supporting for each predicted regulatory interaction. Possible operon structures of the identified regulated genes have not been studied, thus the propagated regulons are still preliminary and need to be improved by operon prediction in the future. Nevertheless, the regulon propagation procedure is considered to be accurate and conservative, since it relies on the manually curated regulons and does not make an attempt for automatic prediction of new members of regulon.

As result, the conservative propagation procedure was applied to 640 genomes from 14 taxonomic groups with available genome-wide collections of reference TF regulogs (Table 2). Three largest taxonomic groups with propagated TF regulons were Enterobacteriales (160 genomes), Bacillales (68 genomes) and Streptococcaceae (69 genomes). The propagated regulons section in RegPrecise 3.0 represents a large set of draft TF regulons annotated in all available genomes within the analyzed taxonomic groups of bacteria (Table 2).

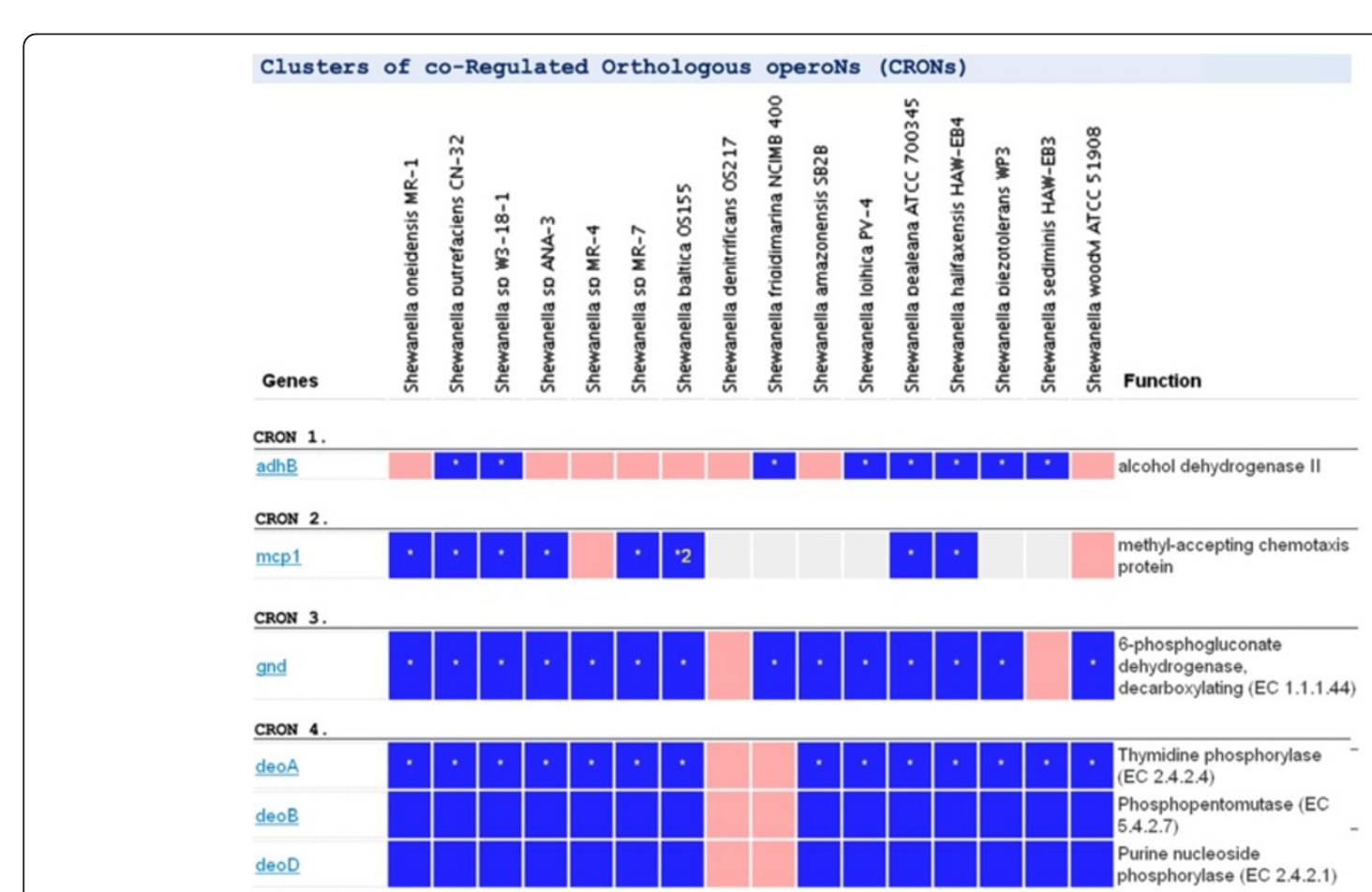

Figure 5 The regulog page in RegPrecise. The screenshot illustrates CRONs constituting the HexR regulog in the Shewanella taxonomic group. 


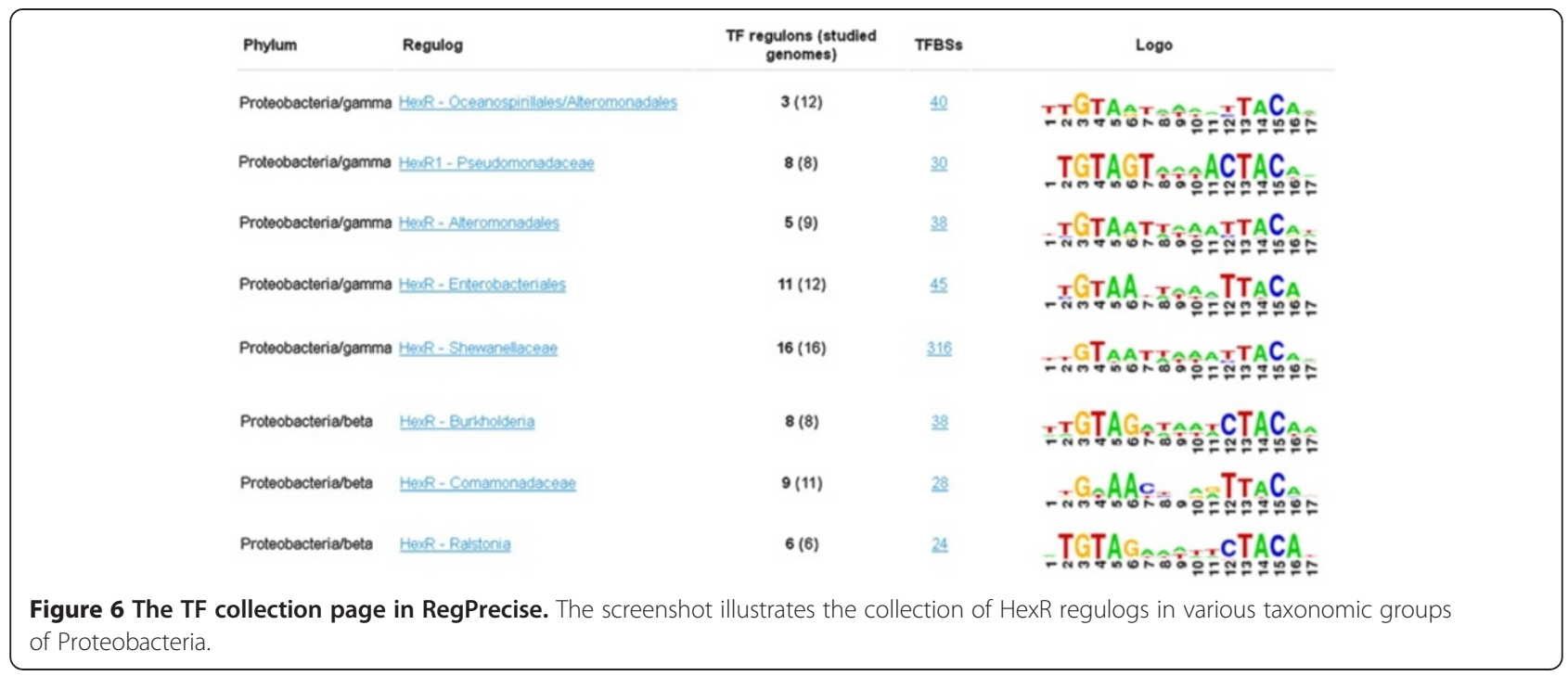

\section{Utility and discussion}

The RegPrecise 3.0 interface provides several different ways to navigate the data. The manually curated reference regulons are accessible via regulog collections and browse web pages, or by using a keyword search tool. The central regulog collections page has entry points to the pages with regulog classifications of six different types: by taxonomic groups, by TFs, by TF families, by
RNA families, by effectors, and by metabolic pathways, as described in the previous section. The browse by regulogs and browse by genomes pages contain the complete lists of all studied regulogs and genomes, and give an alternative way to access each individual regulog and genome page in the database. A keyword search tool located in the top right corner on any RegPrecise page allows a user to search for target genes and regulators using their

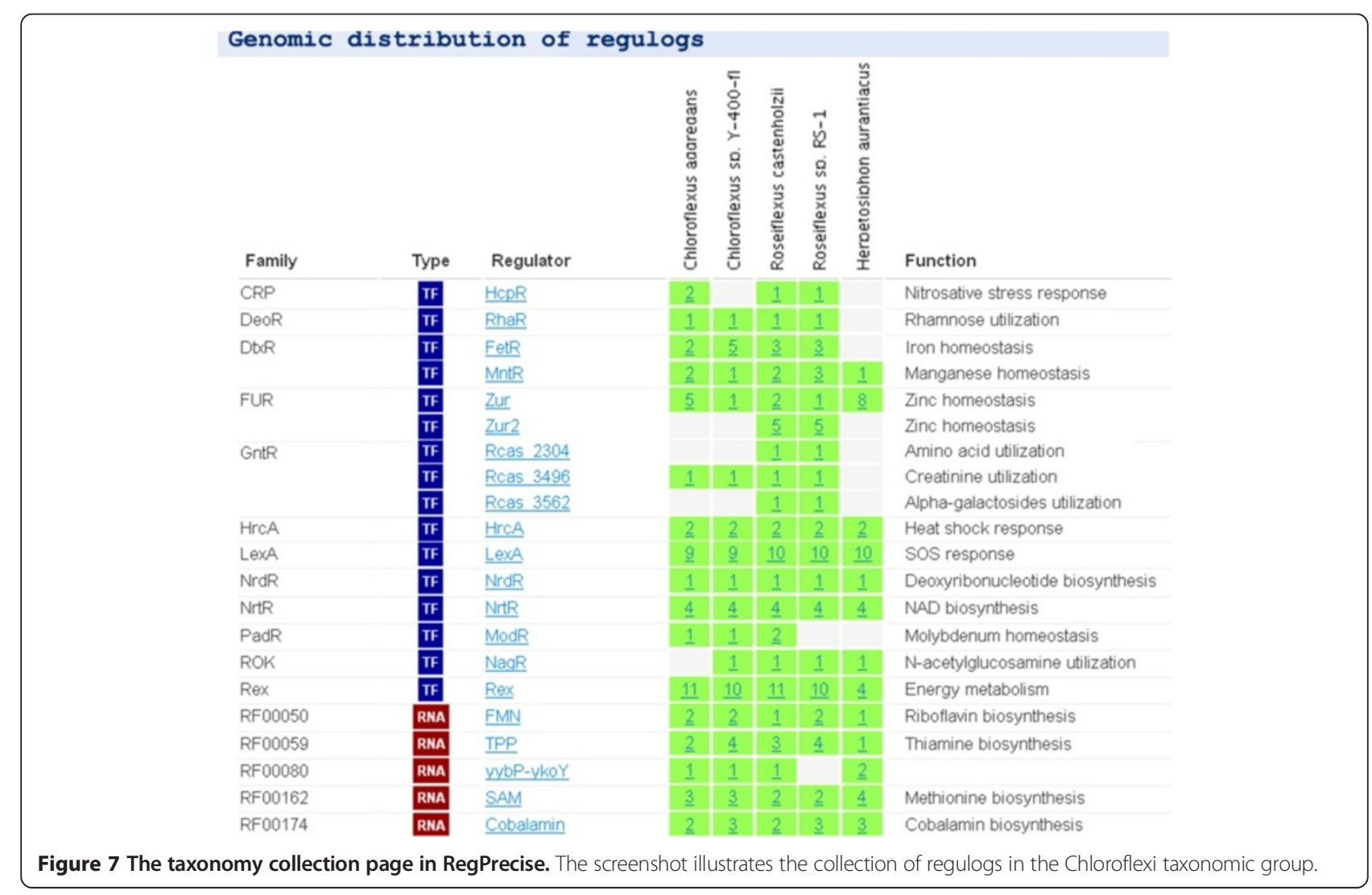


locus tags or names, and to access the corresponding regulon pages for particular genomes. A case study of the HexR regulon below describes major ways to access data and web interfaces in RegPrecise 3.0.

The regulon page (as illustrated by the HexR regulon in Shewanella oneidensis in Figure 3) gives a brief summary for a regulator (TF or RNA type, locus tag, family, regulation mode, regulated biological process, effector) and a complete list of predicted target genes/operons with their locus tags, names and functions. In addition, the regulon page contains cross-links to the parent regulog and regulog collections pages, the TFBS motif page, and the visualization page. The latter page utilizes the GenomeLinked Application for Metabolic Maps (GLAMM) interface and presents the functional overview of a regulon by visualizing its predicted members in the context of metabolic networks [41]. For instance, an image of the HexR regulon in Shewanella shows that it contains genes involved in the energy, carbohydrate and nucleotide metabolisms (Figure 4).

The regulog page allows one to analyze the evolutionary conservation of gene regulation by orthologous regulators in a set of closely related genomes. A comparative table of all CRONs shows a phylogenetic profile of gene regulation by a regulator across the genomes (as illustrated by the HexR regulog in Shewanellaceae in Figure 5). The table of CRONs allows one to identify a core part of the regulog populated by genes with broadly conserved regulatory sites and a variable part of the regulog containing genes with non-conserved sites. The regulog page also has a link to the GLAMM [41] visualization of metabolic content of the entire regulog. For each TF-operated regulog, the TFBS motif logo has a link to the profile page containing detailed information about associated TFBSs (site sequence, score and position relative the gene start).

The collections pages provide access, unique representation, description and summary statistics for regulogs grouped by several properties:

Collections of TFs and TF families (see HexR regulog collection in Figure 6) for each regulog contain unique alignments of TFBSs motif logos, which allow for evolutionary analysis of homologous TFs and their binding motifs.

Collections of RNA families represent and classify all RNA-operated regulons.

Collections of effectors facilitate the analysis of different regulators that respond to the same effector.

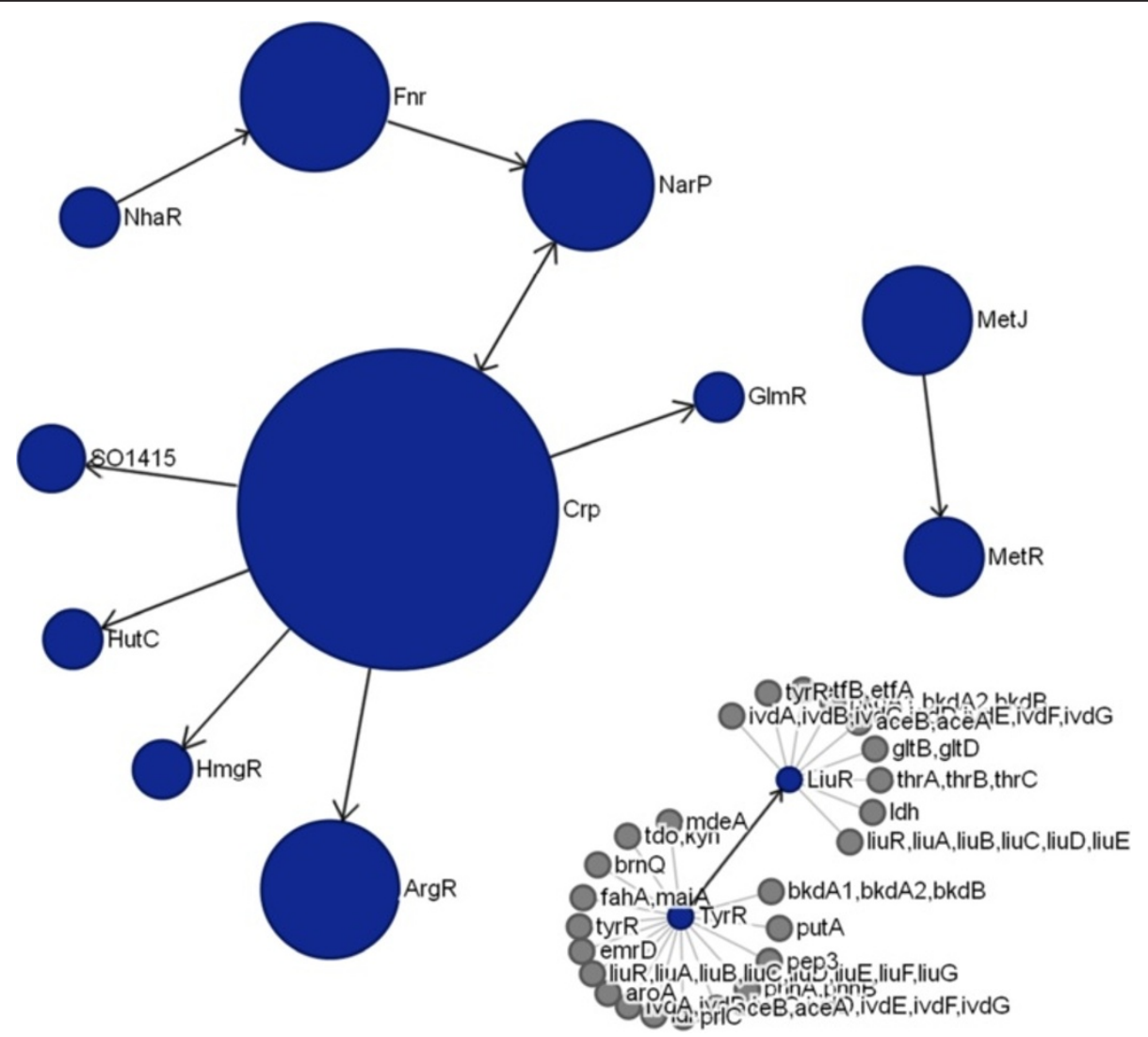

Figure 8 The regulatory cascades on the genome page in RegPrecise. The screenshot illustrates the regulatory cascades in the reconstructed regulatory network of Shewanella oneidensis. 
Collections of pathways identify different regulatory mechanisms for transcriptional control of the same metabolic pathway.

Collections based on taxonomy give an overview for distribution of all reconstructed TF and RNA regulogs arranged by regulog type and family attributes in all analyzed genomes from the same taxonomic group. A taxonomy collection page (see the Chloroflexi regulog collection in Figure 7) highlights universally conserved and narrowly distributed regulogs, and provides crosslinks to both individual regulog and genome pages.

A genome page summarizes information on all reconstructed TF and RNA regulons in a given genome, gives access to a functional overview and visualization of a genome-wide regulatory network. The reconstructed genome-centric TRNs are visualized via an interactive JavaScript widget with scale-up and scale-down functions. Two types of a cross talk between regulons from highly interconnected TRNs are shown as respective page tabs: (i) regulatory cascades (as illustrated for the Shewanella oneidensis TRN in Figure 8), and (ii) coregulations of genes by multiple regulators. Finally, the functional content of an entire TRN in a given genomes is visualized via the GLAMM applet (as illustrated for the Shewanella oneidensis TRN in Figure 9).

The regulon, regulog and TFBS profile pages are linked to relevant datasets of co-regulated genes (as a tabdelimited text) or regulatory sites (as a fasta-formatted text) for export. In addition, the RegPrecise web services interface provides programmatic access to all regulatory interactions and regulon data in the database [22]. All locus tags for TFs and target genes in RegPrecise 3.0 have cross links to web pages in the MicrobesOnline genomic database [39].

We are planning to incorporate supportive experimental evidences for reconstructed regulons and effectors using information from literature and other databases on microbial regulation including RegTransBase [25], RegulonDB [26], DBTBS [27], and CoryneRegNet [24]. We are also planning to develop new graphic modules allowing the RegPredict-style representation of CRONs and species trees on the regulog page. The datasets of reference regulons will be expanded by novel collections for more than 20 taxonomic groups from both Bacteria and Archaea domains.

\section{Conclusions}

The RegPrecise 3.0 is a significantly updated and enhanced version of an open-access database that contains reference collections of curated microbial regulons operated by TFs and RNA and inferred by the comparative genomics. The reference collections of TF regulons from 161 genomes were conservatively propagated to near 500 new genomes. The draft propagated regulons constitute a separate section in the database. RegPrecise provides a unique user-friendly representation of regulatory

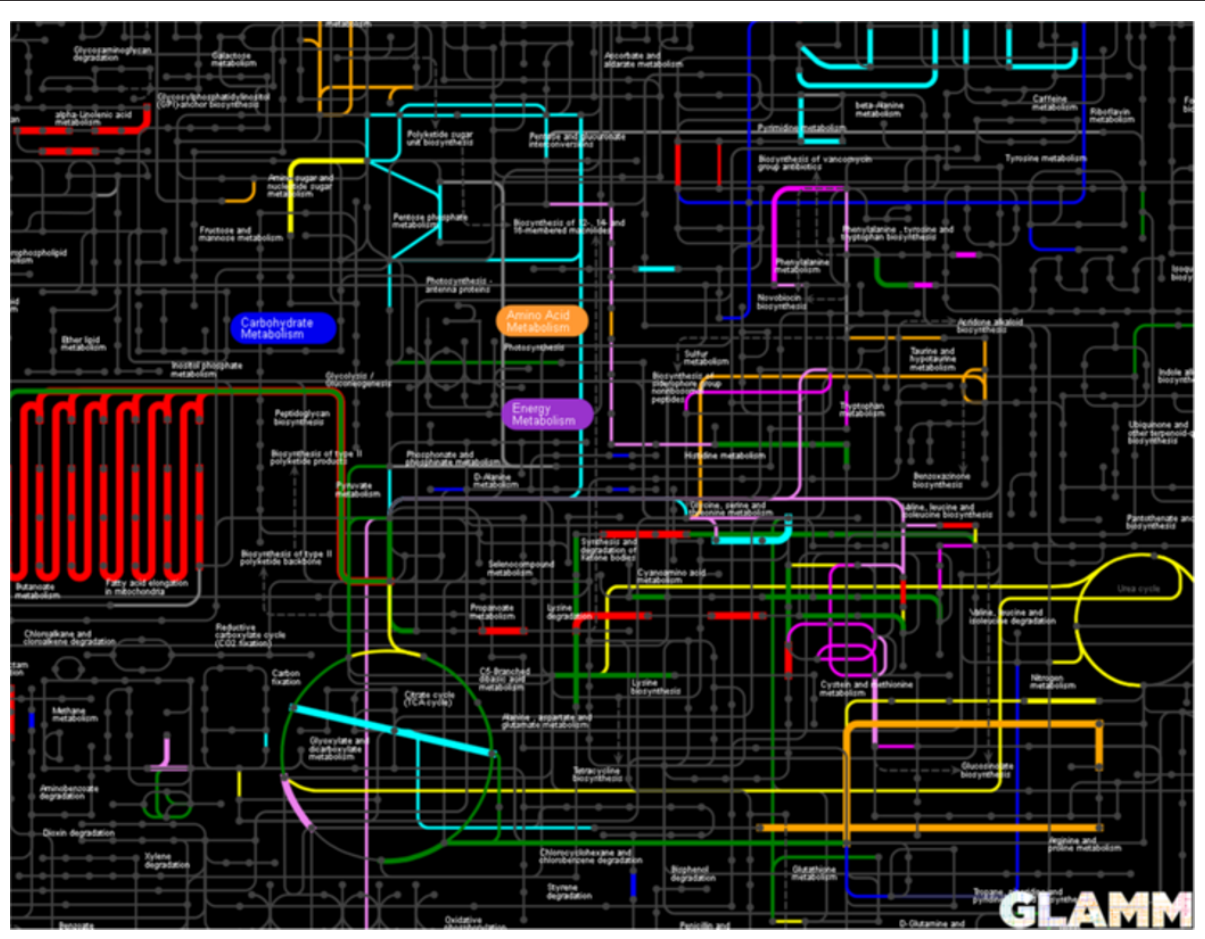

Figure 9 The regulated metabolic pathways on the genome page in RegPrecise. The screenshot illustrates the GLAMM representation of metabolic pathways controlled by all reconstructed regulons in Shewanella oneidensis. 
interactions with multiple interfaces to give access to multiple features of the inferred regulog collections at several hierarchical levels. Accumulated data on the regulatory interactions in diverse bacterial species will be useful for a broad scientific community. In particularly, these data can provide a basis for: 1) planning future experiments for validation of novel regulatory mechanisms inferred by comparative genomics; 2) analyzing evolution of microbial regulatory networks; 3) building predictive biological models combining regulatory and metabolic networks.

\section{Availability and requirements}

RegPrecise 3.0 is freely available at http://regprecise.lbl.gov.

\section{Additional file}

Additional file 1: Domain architectures and protein family classification of TFs in RegPrecise. Each TF family has an assigned domain rule containing known domains from Pfam, COG and Superfamily databases. Text descriptions of TF families include literature references in PubMed.

\section{Abbreviations}

TF: Transcription factor; TFBS: Transcription factor-binding site TRN: Transcriptional regulatory network; PWM: Position weight matrix; CRON: Cluster of co-regulated orthologous operons.

\section{Competing interests}

The author declares that they have no competing interests.

\section{Authors' contributions}

PSN and DARo directed the whole research, designed the database, and wrote the manuscript. PSN developed the database. AEK and GYK developed controlled vocabularies of effectors and pathways. MDK identified riboswitches. AEK, DARo, DARa, and SAL performed comparative genomic analysis to infer transcriptional regulons. DARo curated regulon reconstructions. WR implemented the GLAMM module. RAS implemented regulatory networks visualizations. ID and APA contributed to the design of the study, discussed results and critically revised the manuscript. All the authors have read and approved the final version of the manuscript.

\section{Acknowledgments}

This research was supported by the Genomic Science Program (GSP), Office of Biological and Environmental Research (OBER), U.S. Department of Energy (DOE) under contract DE-SC0004999 with Sanford-Burnham Medical Research Institute (SBMRI) and Lawrence Berkeley National Laboratory (LBNL), the ENIGMA Science Focus Area (SFA) at LBNL (contract DE-AC02-05CH11231), and by the GSP Foundational Science Focus Area (FSFA) of the Pacific Northwest National Laboratory (PNNL). Additional funding was provided by Russian Foundation for Basic Research (12-04-33003, 12-04-32098 and 12-04 31939). MDK was supported by State Contract\#8135 (application 2012-1.2.2 12-000-1013-079)

\footnotetext{
Author details

${ }^{1}$ Lawrence Berkeley National Laboratory, Berkeley 94710, CA, USA. ${ }^{2}$ A.A. Kharkevich Institute for Information Transmission Problems, Russian Academy of Sciences, Moscow 127994, Russia. ${ }^{3}$ Sanford-Burnham Medical Research Institute, La Jolla 92037, CA, USA. ${ }^{4}$ Department of Bioengineering and Bioinformatics, Lomonosov Moscow State University, Leninskye Gory 1-73, Moscow 119992, Russia.
}

Received: 29 June 2013 Accepted: 28 October 2013

Published: 1 November 2013

\section{References}

1. Rodionov DA: Comparative genomic reconstruction of transcriptional regulatory networks in bacteria. Chem Rev 2007, 107(8):3467-3497.

2. Manson McGuire A, Church GM: Predicting regulons and their cis-regulatory motifs by comparative genomics. Nucleic Acids Res 2000, 28(22):4523-4530.

3. McCue L, Thompson W, Carmack C, Ryan MP, Liu JS, Derbyshire V, Lawrence CE: Phylogenetic footprinting of transcription factor binding sites in proteobacterial genomes. Nucleic Acids Res 2001, 29(3):774-782.

4. Mironov AA, Koonin EV, Roytberg MA, Gelfand MS: Computer analysis of transcription regulatory patterns in completely sequenced bacterial genomes. Nucleic Acids Res 1999, 27(14):2981-2989.

5. Tan K, Moreno-Hagelsieb G, Collado-Vides J, Stormo GD: A comparative genomics approach to prediction of new members of regulons. Genome Res 2001, 11(4):566-584.

6. Novichkov PS, Rodionov DA, Stavrovskaya ED, Novichkova ES, Kazakov AE, Gelfand MS, Arkin AP, Mironov AA, Dubchak I: RegPredict: an integrated system for regulon inference in prokaryotes by comparative genomics approach. Nucleic Acids Res 2010, 38:W299-W307.

7. Kazakov AE, Rodionov DA, Alm E, Arkin AP, Dubchak I, Gelfand MS: Comparative genomics of regulation of fatty acid and branched-chain amino acid utilization in proteobacteria. J Bacteriol 2009, 191(1):52-64.

8. Kazakov AE, Rodionov DA, Price MN, Arkin AP, Dubchak I, Novichkov PS: Transcription factor family-based reconstruction of singleton regulons and study of the Crp/Fnr, ArsR, and GntR families in Desulfovibrionales genomes. J Bacteriol 2013, 195(1):29-38.

9. Kazanov MD, Li X, Gelfand MS, Osterman AL, Rodionov DA: Functional diversification of ROK-family transcriptional regulators of sugar catabolism in the Thermotogae phylum. Nucleic Acids Res 2013, 41(2):790-803.

10. Leyn SA, Kazanov MD, Sernova NV, Ermakova EO, Novichkov PS, Rodionov DA: Genomic reconstruction of transcriptional regulatory network in bacillus subtilis. J Bacteriol 2013, 195(11):2463-2473.

11. Leyn SA, Li X, Zheng Q, Novichkov PS, Reed S, Romine MF, Fredrickson JK, Yang C, Osterman AL, Rodionov DA: Control of proteobacterial central carbon metabolism by the HexR transcriptional regulator: a case study in Shewanella oneidensis. J Biol Chem 2011, 286(41):35782-35794.

12. Ravcheev DA, Best AA, Sernova NV, Kazanov MD, Novichkov PS, Rodionov DA: Genomic reconstruction of transcriptional regulatory networks in lactic acid bacteria. BMC Genomics 2013, 14:94.

13. Ravcheev DA, Best AA, Tintle N, Dejongh M, Osterman AL, Novichkov PS, Rodionov DA: Inference of the transcriptional regulatory network in Staphylococcus aureus by integration of experimental and genomicsbased evidence. J Bacteriol 2011, 193(13):3228-3240.

14. Ravcheev DA, Li X, Latif H, Zengler K, Leyn SA, Korostelev YD, Kazakov AE, Novichkov PS, Osterman AL, Rodionov DA: Transcriptional regulation of central carbon and energy metabolism in bacteria by redox-responsive repressor Rex. J Bacteriol 2012, 194(5):1145-1157.

15. Rodionov DA, Dubchak I, Arkin A, Alm E, Gelfand MS: Reconstruction of regulatory and metabolic pathways in metal-reducing delta-proteobacteria. Genome Biol 2004, 5(11):R90.

16. Rodionov DA, Dubchak IL, Arkin AP, Alm EJ, Gelfand MS: Dissimilatory metabolism of nitrogen oxides in bacteria: comparative reconstruction of transcriptional networks. PLoS Comput Biol 2005, 1(5):e55.

17. Rodionov DA, Gelfand MS: Identification of a bacterial regulatory system for ribonucleotide reductases by phylogenetic profiling. Trends Genet 2005, 21(7):385-389.

18. Rodionov DA, Gelfand MS, Todd JD, Curson AR, Johnston AW: Computational reconstruction of iron- and manganese-responsive transcriptional networks in alpha-proteobacteria. PLoS Comput Biol 2006, 2(12):e163.

19. Rodionov DA, Novichkov PS, Stavrovskaya ED, Rodionova IA, Li X, Kazanov MD, Ravcheev DA, Gerasimova AV, Kazakov AE, Kovaleva GY, et al: Comparative genomic reconstruction of transcriptional networks controlling central metabolism in the Shewanella genus. BMC Genomics 2011, 12(Suppl 1):S3.

20. Rodionov DA, Rodionova IA, Li X, Ravcheev DA, Tarasova Y, Portnoy VA, Zengler $\mathrm{K}$, Osterman AL: Transcriptional regulation of the carbohydrate utilization network in Thermotoga maritima. Frontiers in microbiology 2013, 4:244.

21. Novichkov PS, Laikova ON, Novichkova ES, Gelfand MS, Arkin AP, Dubchak I, Rodionov DA: RegPrecise: a database of curated genomic inferences of transcriptional regulatory interactions in prokaryotes. Nucleic Acids Res 2010, 38:D111-D118. Database issue.

22. Novichkov PS, Brettin TS, Novichkova ES, Dehal PS, Arkin AP, Dubchak I, Rodionov DA: RegPrecise web services interface: programmatic access to the 
transcriptional regulatory interactions in bacteria reconstructed by comparative genomics. Nucleic Acids Res 2012, 40:W604-W608. Web Server issue.

23. Sun El, Leyn SA, Kazanov MD, Saier MH, Novichkov PS, Rodionov DA: Comparative genomics of metabolic capacities of regulons controlled by cis-regulatory RNA motifs in bacteria. BMC Genomics 2013, 14(1):597.

24. Baumbach J: CoryneRegNet 4.0 - A reference database for corynebacterial gene regulatory networks. BMC Bioinforma 2007, 8:429.

25. Cipriano MJ, Novichkov PN, Kazakov AE, Rodionov DA, Arkin AP, Gelfand MS, Dubchak I: RegTransBase - a database of regulatory sequences and interactions based on literature: a resource for investigating transcriptional regulation in prokaryotes. BMC Genomics 2013, 14(1):213.

26. Gama-Castro S, Jimenez-Jacinto V, Peralta-Gil M, Santos-Zavaleta A, Penaloza-Spinola MI, Contreras-Moreira B, Segura-Salazar J, Muniz-Rascado L, Martinez-Flores I, Salgado H, et al: RegulonDB (version 6.0): gene regulation model of Escherichia coli K-12 beyond transcription, active (experimental) annotated promoters and Textpresso navigation. Nucleic Acids Res 2008, 36:D120-D124. Database issue.

27. Sierro N, Makita Y, de Hoon M, Nakai K: DBTBS: a database of transcriptional regulation in Bacillus subtilis containing upstream intergenic conservation information. Nucleic Acids Res 2008, 36:D93-D96. Database issue.

28. Gardner PP, Daub J, Tate JG, Nawrocki EP, Kolbe DL, Lindgreen S, Wilkinson AC, Finn RD, Griffiths-Jones S, Eddy SR, et al: Rfam: updates to the RNA families database. Nucleic Acids Res 2009, 37:D136-D140. Database issue.

29. Nawrocki EP, Kolbe DL, Eddy SR: Infernal 1.0: inference of RNA alignments. Bioinformatics 2009, 25(10):1335-1337.

30. Rodionov DA, De Ingeniis J, Mancini C, Cimadamore F, Zhang H, Osterman AL, Raffaelli N: Transcriptional regulation of NAD metabolism in bacteria: NrtR family of Nudix-related regulators. Nucleic Acids Res 2008, 36(6):2047-2059.

31. Rodionov DA, Li X, Rodionova IA, Yang C, Sorci L, Dervyn E, Martynowski D, Zhang H, Gelfand MS, Osterman AL: Transcriptional regulation of NAD metabolism in bacteria: genomic reconstruction of NiaR (YrXA) regulon. Nucleic Acids Res 2008, 36(6):2032-2046.

32. Suvorova IA, Tutukina MN, Ravcheev DA, Rodionov DA, Ozoline ON, Gelfand MS: Comparative genomic analysis of the hexuronate metabolism genes and their regulation in gammaproteobacteria. J Bacteriol 2011, 193(15):3956-3963.

33. Rodionov DA, Mironov AA, Gelfand MS: Transcriptional regulation of pentose utilisation systems in the Bacillus/Clostridium group of bacteria. FEMS Microbiol Lett 2001, 205(2):305-314.

34. Rodionov DA, Mironov AA, Gelfand MS: Conservation of the biotin regulon and the BirA regulatory signal in Eubacteria and Archaea. Genome Res 2002, 12(10):1507-1516.

35. Chen X, Kohl TA, Ruckert C, Rodionov DA, Li LH, Ding JY, Kalinowski J, Liu SJ: Phenylacetic acid catabolism and its transcriptional regulation in Corynebacterium glutamicum. Appl Environ Microbiol 2012, 78(16):5796-5804.

36. Punta M, Coggill PC, Eberhardt RY, Mistry J, Tate J, Boursnell C, Pang N, Forslund K, Ceric G, Clements J, et al: The Pfam protein families database. Nucleic Acids Res 2012, 40:D290-D301. Database issue.

37. Tatusov RL, Fedorova ND, Jackson JD, Jacobs AR, Kiryutin B, Koonin EV, Krylov DM, Mazumder R, Mekhedov SL, Nikolskaya AN, et al: The COG database: an updated version includes eukaryotes. BMC Bioinforma 2003, 4:41.

38. Wilson D, Pethica R, Zhou Y, Talbot C, Vogel C, Madera M, Chothia C, Gough J: SUPERFAMILY-sophisticated comparative genomics, data mining, visualization and phylogeny. Nucleic Acids Res 2009, 37:D380-386. Database issue.

39. Dehal PS, Joachimiak MP, Price MN, Bates JT, Baumohl JK, Chivian D, Friedland GD, Huang KH, Keller K, Novichkov PS, et al: MicrobesOnline: an integrated portal for comparative and functional genomics. Nucleic Acids Res 2010, 38:D396-D400. Database issue.

40. Overbeek R, Begley T, Butler RM, Choudhuri JV, Chuang HY, Cohoon M, de Crecy-Lagard V, Diaz N, Disz T, Edwards R, et al: The subsystems approach to genome annotation and its use in the project to annotate 1000 genomes. Nucleic Acids Res 2005, 33(17):5691-5702.

41. Bates JT, Chivian D, Arkin AP: GLAMM: Genome-Linked Application for Metabolic Maps. Nucleic Acids Res 2011, 39:W400-W405. Web Server issue.

doi:10.1186/1471-2164-14-745

Cite this article as: Novichkov et al:: RegPrecise 3.0 - A resource for genome-scale exploration of transcriptional regulation in bacteria. $B M C$ Genomics 2013 14:745.

\section{Submit your next manuscript to BioMed Central and take full advantage of:}

- Convenient online submission

- Thorough peer review

- No space constraints or color figure charges

- Immediate publication on acceptance

- Inclusion in PubMed, CAS, Scopus and Google Scholar

- Research which is freely available for redistribution 